\title{
CoRot observation of a young Sun-like star
}

\author{
P. Gondoin ${ }^{1}$, D. Gandolfi ${ }^{1}$, M. Fridlund ${ }^{1}$, \\ E. W. Guenther ${ }^{2}$ and A. Hatzes ${ }^{2}$
}

${ }^{1}$ European Space Agency, ESTEC - Postbus 299, 2200 AG Noordwijk, The Netherlands

${ }^{2}$ Thüringer Landessternwarte Tautenburg, Sternwarte 5, 07778 Tautenburg, Germany

\begin{abstract}
The present study reports measurements of the rotation period of a young solar analogue, estimates of its surface coverage by photospheric starspots and of its chromospheric activity level, and derivations of its evolutionary status. It compares the chromospheric activity level of this young star with a model of chromospheric activity evolution established by combining relationships between the $R_{\mathrm{HK}}^{\prime}$ index and the Rossby number with a recent model of stellar rotation evolution on the main-sequence.
\end{abstract}

Keywords. stars: activity, stars: atmospheres, stars: late-type, stars: magnetic fields, stars: rotation, stars: spots.

We analysed the high-accuracy CoRot light curve of the active star CoRoT 102899501 using a model based on the rotational modulation of the visibility of active regions (Fig. 1). The analysis results suggest that the spot coverage of its surface increase as a function of time from 5-14 \% at the beginning of the observing run to $13-29 \% 35$ days later. The starspots used as tracers of the star rotation constrain the rotation period to $1.625 \pm 0.002$ days and do not show evidence for differential rotation.

A high level of magnetic activity on Corot 102899501 is corroborated by a strong emission in the Balmer and Ca II H\& K lines $\left(\log R_{\mathrm{HK}}^{\prime} \sim-4\right)$ (Fig. 2). The effective temperature $\left(T_{\text {eff }}=5180 \pm 80 \mathrm{~K}\right)$, surface gravity $(\log g=4.35 \pm 0.1)$, and metallicity $([\mathrm{M} / \mathrm{H}]=0.05 \pm 0.07 \mathrm{dex})$ indicate that the object is located near the evolutionary track of a $1.09 \pm 0.12 \mathrm{M}_{\odot}$ pre-main sequence star at an age of $23 \pm 10$ Myrs.

We compared (Fig. 3) the chromospheric activity level of this young star with a model of chromospheric activity evolution (Gondoin et al. 2012) established by combining relationships between the $R_{\mathrm{HK}}^{\prime}$ index and the Rossby number (Mamajek \& Hillenbrand 2008 ) with a recent model of stellar rotation evolution on the main-sequence (Barnes 2010).

We conclude that the high-magnetic activity level and the fast rotation of the observed star are manifestation of its stellar youth consistent with its estimated evolutionary status

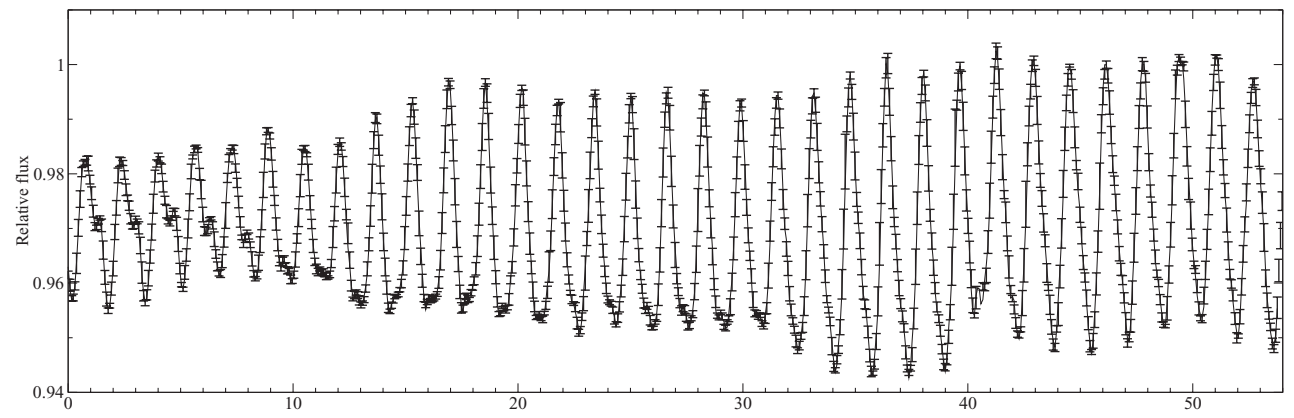

Figure 1. Best fit model to the light-curve of CoRoT 102899501. 


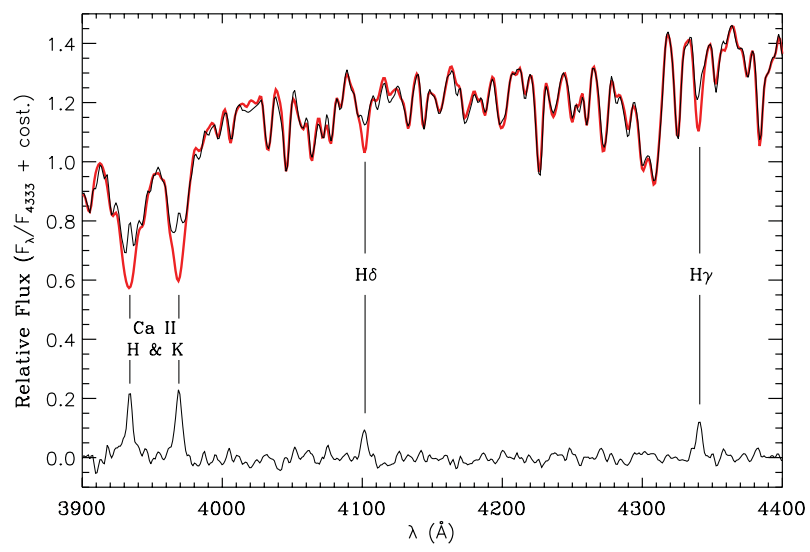

Figure 2. Spectrum of CoRoT 102899501 along with the best-fitting template overplotted.

and with the detection of a strong Li I $\lambda 6707.8 \AA$ absorption line in its spectrum. We argue that a magnetic activity level comparable to that observed on CoRot 102899501 could have been present on the Sun at the time of planet formation.

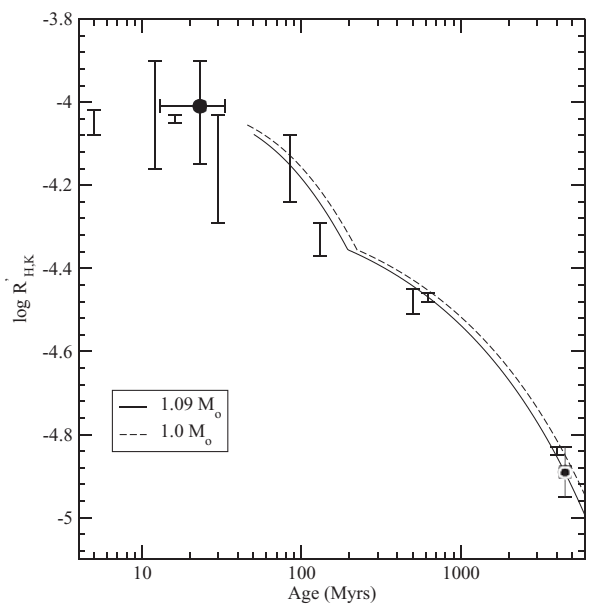

Figure 3. Time evolution model of the chromospheric activity of Sun-like stars on the main-sequence compared with the median chromospheric activity indices of the Sun and solar-type dwarfs in Upper Sco (age $\sim 5$ Myrs), $\beta$ Pic ( 12 Myrs), Upper Cen-Lup ( 16 Myrs), Lower Cen Cru $(\sim 16$ Myrs $), \alpha$ Per $(\sim 85$ Myrs $)$, the Pleiades $(\sim 130$ Myrs $)$, UMa $(\sim 500$ Myrs $)$, the Hyades ( $\sim 625$ Myrs), and M67 ( 4000 Myrs). The value of the chromospheric activity index $\log \left(R_{\mathrm{HK}}^{\prime}\right)$ of CoRoT 102899501 is indicated with a black circle.

\section{References}

Barnes, S. A. 2010, ApJ, 722, 222

Gondoin, P., Gandolfi, D., Fridlund M. et al. 2012, A\&A, accepted

Mamajek, E. E. \& Hillenbrand, L. A. 2008, ApJ, 687, 1264 